\title{
Féeries
}

Études sur le conte merveilleux, XVII $-\mathrm{XIXe}$ siècle

$14 \mid 2017$

Conte merveilleux et poésie

\section{Imagination scientifique et littérature merveilleuse, Charles Tiphaigne de La Roche}

textes recueillis et présentés par Yves Citton, Marianne Dubacq et Philippe Vincent, Presses universitaires de Bordeaux, coll. « Mirabilia », 2014

Régine Jomand-Baudry

\section{OpenEdition}

Édition électronique

URL : http://journals.openedition.org/feeries/1175

DOI : $10.4000 /$ feeries. 1175

ISSN : 1957-7753

Éditeur

UGA Éditions/Université Grenoble Alpes

Édition imprimée

ISBN : 978-2-37747-012-9

ISSN : $1766-2842$

Référence électronique

Régine Jomand-Baudry, « Imagination scientifique et littérature merveilleuse, Charles Tiphaigne de La Roche », Féeries [En ligne], 14 | 2017, mis en ligne le 31 juillet 2017, consulté le 24 septembre 2020. URL : http://journals.openedition.org/feeries/1175; DOI : https://doi.org/10.4000/feeries. 1175

Ce document a été généré automatiquement le 24 septembre 2020

(c) Féeries 


\section{Imagination scientifique et littérature merveilleuse, Charles Tiphaigne de La Roche}

textes recueillis et présentés par Yves Citton, Marianne Dubacq et Philippe Vincent, Presses universitaires de Bordeaux, coll. " Mirabilia », 2014

Régine Jomand-Baudry

\section{RÉFÉRENCE}

Imagination scientifique et littérature merveilleuse, Charles Tiphaigne de La Roche, textes recueillis et présentés par Yves Citton, Marianne Dubacq et Philippe Vincent, Presses universitaires de Bordeaux, coll. « Mirabilia », 2014

1 Deux ouvrages parus ces dernières années nous invitent à découvrir ou à redécouvrir le curieux auteur que fut Charles François Tiphaigne de La Roche dont l'œuvre se situe au croisement de la science et du merveilleux. Le premier est la version remaniée et complétée d'un mémoire de DEA soutenu par Philippe Vincent à l'université Jean Moulin-Lyon 3, édité par les Publications des universités de Rouen et du Havre (2012): il s'agit de l'édition critique de la plus célèbre fantaisie scientifique de Tiphaigne, et sans doute de la plus séduisante: Amilec ou la graine d'hommes, qui se présente comme une mise en fiction des théories de la génération. Le second, intitulé Imagination scientifique et littérature merveilleuse, fournit les actes d'un colloque organisé par Yves Citton et l'UMR LIRE à l'université Stendhal-Grenoble 3 (2010), exclusivement consacré à l'œuvre du médecin normand. Il est publié aux Presses universitaires de Bordeaux dans la collection « Mirabilia » (2014). Ce regain d'activité autour d'un auteur jusque-là largement oublié - citons plusieurs travaux d'Yves Citton et une publication des Euvres complètes du médecin normand en cours sous la direction de Jacques Marx s'explique doublement aux yeux des auteurs: non seulement par l'actualité de la 
réflexion de Tiphaigne, notamment sur des questions environnementales, mais aussi parce que cet auteur choisit, pour éprouver sa pensée au frottement des savoirs de ses contemporains, le merveilleux ou l'onirisme scientifique et la stratégie d'une écriture bigarrée.

Dans une première partie, ce recueil repose, dans le sillage des travaux inauguraux de Jacques Marx, la question de la place de Tiphaigne dans les débats philosophiques et littéraires de son époque : est-il, comme une certaine tradition l'établit, un marginal à la pensée éparpillée, puisant aussi bien dans la philosophie occulte que dans les théories qui lui sont contemporaines? Les écrits de celui qu'on a pu nommer un «magicien de la raison", s'ils héritent de la tradition hermétique, sont surtout au carrefour des idées de son temps, qu'il s'agisse du vitalisme ou de la philosophie expérimentale, à la source des fictions les plus merveilleusement surprenantes et les plus novatrices (Jacques Marx). La réception de l'œuvre de Tiphaigne dans la presse contemporaine confirme d'ailleurs cette tension, particulièrement sensible dans les comptes rendus d'Amilec et de Giphantie, lus alternativement à travers les filtres de la philosophie et de l'antiphilosophie: c'est souvent l'audace de la pensée de Tiphaigne qui est relevée, et masquée derrière la bizarrerie de la fiction littéraire, l'énoncé de vérités autrement inaudibles. Ph. Vincent, tout en faisant le compte minutieux des œuvres à attribuer de manière certaine à Tiphaigne, s'attache à montrer derrière l'apparente bigarrure, la cohérence d'une œuvre caractérisée sur le plan des idées par un scepticisme envers les systèmes, par une conception de la physique de l'amour reconnaissant l'action de la matière sympathique sur les organes des sens et par la croyance en l'immatérialité de l'âme; de même, si, au plan générique, l'auteur cultive le disparate, c'est pour mieux refuser l'enfermement dans un genre, et s'il place son œuvre sous le signe de la polyphonie, c'est pour garantir le dialogue entre une multiplicité de voix.

3 Une seconde partie s'interroge tout naturellement sur le travail de l'écriture et de la pensée. Pour Michèle Bokobza-Kahan, qui étudie dans le même temps la position de l'auteur dans le champ littéraire et le mode d'inscription d'une image auctoriale de soi dans le texte de fiction, tous les indices convergent pour faire de Tiphaigne un écrivain de la marginalité : esquives de l'auctorialité manifeste dans les préfaces, méfiance à l'égard de toute activité intellectuelle, doublée d'un mépris vis-à-vis des écrivains et des philosophes dont il dénonce la soumission aux lois du nouveau marché littéraire, construisent une identité éditoriale clivée. Témoignent également de cette tension : le mélange des genres et la conjugaison déroutante de l'écriture scientifique et de l'écriture fictionnelle, les procédés d'effacement de l'instance auctoriale manifestes dans l'hétérogénéité discursive et les mises en place particulières du discours rapporté. Ces procédés font de Tiphaigne le champion de la distanciation critique et du scepticisme ironique, et l'ennemi de toute position dogmatique. "Singulier", «original», «bizarre», tous ces adjectifs que l'on retrouve dans la critique contemporaine pour qualifier l'œuvre ou l'auteur, Florence Boulerie se propose de les appréhender à partir du « loufoque ", qu'il soit « une façon toute intuitive de réfléchir » ou une forme de futilité. Ce qui est remarquable et dérangeant chez Tiphaigne et ce qui fait l'unité de son œuvre, c'est que l'on retrouve dans la fiction utopique, comme dans l'essai, les mêmes thèmes et les mêmes formes discursives - ainsi la dissertation oscillant entre esprit de sérieux et rire libérateur de la pensée. En s'intéressant plus particulièrement au rapsodique Voyage aux limbes, Julie Boch démontre comment 
Tiphaigne y dénonce en fait une double vanité : celle du savoir scientifique et celle de la philosophie, tous deux impuissants à comprendre le monde physique. Seule la vanité considérée sous l'angle moral trouve paradoxalement (et ironiquement?) grâce aux yeux de l'auteur, car elle est capable de nous orienter dans notre recherche du bonheur. Jean-François Perrin aborde la question de la position philosophique de Tiphaigne au sein des querelles idéologiques du temps en s'attachant aux Visions d'Ibraïm, et spécialement à la question de la nature physiologique et ontologique des songes. Il y démontre à quel point Tiphaigne connaît à la perfection les théories des philosophes sur ce sujet et surtout que, loin d'être un antiphilosophe patenté, il développe une pensée singulière qui ne permet pas de le ranger définitivement au nombre des sceptiques. Il reste à la fois partisan d'une métaphysique dualiste - ce qui le rapproche de Rousseau -, tout en dénonçant les «illusions des Encyclopédistes » à propos «de la thérapeutique d'éducation collective qu'ils engagent». Derrière la poétique du désordre, c'est bien les apories de la pensée moderne qui sont pointées.

Une troisième partie réunit des contributions qui pensent la dimension la plus caractéristique de l'écriture de Tiphaigne, celle qui retiendra particulièrement l'attention des lecteurs de Féeries: le croisement récurrent entre science et merveille. Aurélia Gaillard pose le problème de cet entrelacement sous un angle original, « celui du pacte de lecture et de la méthode scientifique qu'il détermine", non sans avoir redéfini le rapport entre science et merveilleux tel qu'il apparaît chez Fontenelle où le merveilleux émane de la connaissance et non plus de l'ignorance. La lecture attentive de trois des œuvres de Tiphaigne (L'Amour dévoilé, Amilec et Histoire des Galligènes) montre que ce qui est en jeu dans l'écriture de l'auteur, c'est l'équivalence, eu égard à la vérité, du discours de la science et de celui de la fable comme mode de représentation de la nature, et une mise en question de «la procédure de légitimation scientifique ». Ainsi peut-on figurer le monde à sa guise et faire œuvre artistique, que l'on soit mathématicien ou poète. De son côté, Guilhem Armand interprète le mélange de la science et du merveilleux de l'écriture de Tiphaigne comme une volonté de montrer «la vocation fantaisiste de la science» et non pas, comme ses prédécesseurs, «la vocation scientifique de la fiction $"$ : puisque les discours scientifiques sont inaptes à dire la vérité du monde, pourquoi ne pas prendre la liberté de proposer, pour le plaisir de l'émerveillement, un autre monde ? C'est l'option choisie par Tiphaigne qui combine habilement dans son argumentaire les théories sur la génération des dix années précédentes. Chez lui, l'imbrication étroite entre science et merveille est telle qu'elle ne permet pas de savoir laquelle est à l'origine de l'autre. Il invente ainsi un merveilleux moderne en conférant à l'objet merveilleux « une sorte de continuité avec l'univers réel de référence ». La contribution d'Emmanuelle Sempère, centrée sur les images et la pensée du végétal, apporte une conclusion synthétique à cette partie : s'appuyant sur une analyse fine, elle rappelle combien pour Tiphaigne le végétal est « objet de science - au sens de discours scientifique sans totalisation - et de fiction ». Et si "puissance poétique de cet imaginaire du végétal » il y a, elle est à chercher du côté d'un processus à double détente propre à ouvrir aux lecteurs les horizons du rêve: l'absorption des "images littéraires et scientifiques", d'une somme de savoirs de tous ordres, et un mouvement de recréation original et exubérant.

Dans la quatrième partie intitulée "Les ambivalences du merveilleux moderne ", c'est la modernité des inventions les plus loufoques de Tiphaigne qui est mise en avant. Confrontant la mise en scène " d'entités invisibles » dans L'Empire des Zaziris et le Roman cabalistique (1750), et leur pouvoir d'action sur les instincts des individus, Yves Citton 
voit dans les attributs ambivalents accordés par Tiphaigne à ses esprits doués d'une incomparable clairvoyance " une vision anticipée des dispositifs de surveillance globale qui caractérisent notre modernité tardive ». Comme l'auteur du Roman cabalistique, Tiphaigne construit une véritable «politique de la merveille » dont la caractéristique est à la fois d'induire la croyance tout en en dénonçant l'extravagance. Carmen Ramirez s'intéresse quant à elle aux sources de L'Amour dévoilé pour définir le merveilleux de Tiphaigne dans la lignée du baroque dont il adopte nombre de caractéristiques : jeux de miroirs, labyrinthe des voix narratives, complexité des références et des images constituent un merveilleux paradoxal nourri de savoir et suscitant la curiosité. Ce merveilleux, Carmen Ramirez propose de le nommer un "merveilleux mélancolique » en ce qu'il réconcilie l'étendue de l'imagination auctoriale et la nostalgie sans illusion que l'on relève dans la satire. C'est la figure du papillon, par son attrait sensuel et son évanescence, par la fascination dont il fait l'objet du côté des hommes de science, qui cristallise les différents aspects de cet imaginaire.

6 S'il est une image que Tiphaigne a laissée à notre modernité, c'est bien celle d'un inventeur prophétique en matière technologique, puisque dans certains passages anticipateurs, il décrit des « machines » qu'il nous est aisé de reconnaitre. Ce sont à ces merveilles technologiques et à sa qualité de précurseur que s'attachent les deux dernières contributions. Yoshiko Terao et Denis Reynaud replacent sa fameuse "invention" fictive de la photographie dans le développement des "machines à images » telles qu'on peut les appréhender dans la fiction et les ouvrages techniques du temps, et au sein même de la fiction de la Giphantie, qui présente également un dispositif acoustique permettant d'entendre ce qui se dit à un point donné de la Terre. Il s'agit cependant moins pour l'auteur de satisfaire des ambitions humaines que de révéler leur futilité et leur vanité. Replaçant Tiphaigne dans les cadres de pensée de son époque où machinisme et biologie sont perçus dans une sorte de continuité, Isabella Mattazzi met au jour la dimension proprement mécanique du psychisme humain, tel que le conçoit l'auteur : il s'agit bien, par l'invention d'images propres à faire percevoir l'invisible, d'une investigation des modalités de nos expériences mentales. Y. Citton conclut en soulignant le double intérêt de ces «mirabilia " que sont les écrits de Tiphaigne : certes, leur caractère atypique et inclassable n'en finit pas d'étonner, mais surtout, en permettant de relire l'ambivalence de notre modernité avec d'autres modes de pensée, elles ouvrent sur ce qu'il nomme une «altermodernité » dont il décline les différentes dimensions. L'ouvrage se clôt par une annexe de Françoise Weil sur Tiphaigne et le monde de la librairie : le classement de ses ouvrages montre qu'un seul a bénéficié d'un privilège, cinq d'une permission tacite et trois ont été publiés sans permission. Remercions les auteurs de cette somme de réflexions qui nous fait pénétrer au fondement d'un imaginaire aussi insolite que novateur. 


\section{AUTEUR}

RÉGINE JOMAND-BAUDRY

Université Jean Moulin - Lyon 3 